\title{
Deformations of surface defect moduli spaces
}

\author{
Andrew Neitzke ${ }^{a}$ and Ali Shehper ${ }^{b}$ \\ ${ }^{a}$ Department of Mathematics, Yale University, \\ 10 Hillhouse Avenue, New Haven, CT 06511, U.S.A. \\ ${ }^{b}$ Department of Physics, University of Texas at Austin, \\ 2515 Speedway, Austin, TX 78712, U.S.A. \\ E-mail: andrew.neitzke@yale.edu, shehper@utexas.edu
}

ABstRACT: Given a $4 \mathrm{~d} \mathcal{N}=2$ superconformal theory with an $\mathcal{N}=(2,2)$ superconformal surface defect, a marginal perturbation of the bulk theory induces a complex structure deformation of the defect moduli space. We describe a concrete way of computing this deformation using the bulk-defect OPE.

Keywords: Conformal Field Theory, Supersymmetric Gauge Theory, Field Theories in Lower Dimensions

ArXiv EPrint: 2011.01970 


\section{Contents}

1 Introduction 1

2 Properties of $\mathbf{2 d - 4 d}$ systems 5

2.1 Superconformal symmetry of 2d-4d systems 5

2.2 Descendants of superconformal primaries 6

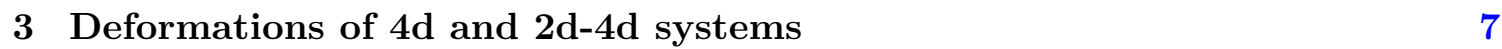

3.1 Deformation of pure 4d systems

3.2 Deformations of 2d-4d systems 8

3.3 Deformations of the supercurrent 9

3.4 Mixing between chiral and anti-chiral descendants 10

$\begin{array}{ll}3.5 & \text { The complex structure deformation } \\ \end{array}$

4 Example: U(1) gauge theory with solenoid defect 14

$\begin{array}{lll}4.1 & \text { Setup } & 14\end{array}$

$\begin{array}{lll}4.2 & \text { Complex deformation } & 15\end{array}$

A Four-dimensional superconformal algebra 15

$\begin{array}{ll}\text { B Two-dimensional superconformal algebra } & 17\end{array}$

$\begin{array}{ll}\text { C Deformations of complex manifolds } & 17\end{array}$

$\begin{array}{ll}\text { D The covariant derivative of the supercurrent } & 18\end{array}$

$\begin{array}{ll}\text { D.1 Computation } & 18\end{array}$

D.2 An integral 20

D.3 With a surface defect 21

\section{Introduction}

It is by now a well known principle that to get a complete picture of a quantum field theory one should study not only local operators, but more generally defects of all dimensions. These defects give rise to rich algebraic, topological and geometric structures. A deformation of the quantum field theory then must induce deformations of all of these structures, fitting together in a self-consistent way, which may be rather intricate in its full generality.

This paper concerns a small part of that story. Given an $\mathcal{N}=2$ superconformal quantum field theory in four dimensions, and an $\mathcal{N}=(2,2)$ superconformal surface defect, we ask, how does a deformation of the theory induce a deformation of the complex structure of the surface defect moduli space? 
In the rest of this introduction we describe this problem more carefully and formulate our proposed answer.

Bulk and defect moduli spaces for $4 \mathrm{~d} \mathcal{N}=\mathbf{2}$ theories. Suppose we are given a $d=4, \mathcal{N}=2$ superconformal theory. The theory has a moduli space $\mathcal{M}_{4 \mathrm{~d}}$ of marginal supersymmetric couplings, which is naturally complex. Now we introduce a $\frac{1}{2}$-BPS surface defect preserving $d=2, \mathcal{N}=(2,2)$ superconformal symmetry [1, 2]. Holding the $4 \mathrm{~d}$ coupling $\tau \in \mathcal{M}_{4 \mathrm{~d}}$ fixed, such a surface defect has a moduli space $\mathcal{M}_{2 \mathrm{~d}}$ of marginal chiral deformations, which is again a complex manifold.

Letting both couplings vary, we have a combined $2 \mathrm{~d}-4 \mathrm{~d}$ moduli space $\mathcal{M}_{2 \mathrm{~d}-4 \mathrm{~d}}$ which is a holomorphic fiber bundle over $\mathcal{M}_{4 \mathrm{~d}}$. As we vary $\tau \in \mathcal{M}_{4 \mathrm{~d}}$, the complex structure of the fiber $\mathcal{M}_{2 \mathrm{~d}}$ may in general vary. The infinitesimal version of this statement is that there is a linear map

$$
T \mathcal{M}_{4 \mathrm{~d}} \rightarrow \operatorname{Def}\left(\mathcal{M}_{2 \mathrm{~d}}\right)
$$

where $\operatorname{Def}\left(\mathcal{M}_{2 \mathrm{~d}}\right)$ means the space of linearized deformations of the complex structure of $\mathcal{M}_{2 \mathrm{~d}}$.

The case of class $\boldsymbol{S}$. On abstract grounds we know that the map (1.1) exists, but one might wonder whether this map could be zero. Indeed in some examples it will be zero (e.g. it must be zero if we study a "surface defect" which is just a $d=2, \mathcal{N}=(2,2)$ theory uncoupled from the bulk theory!) Still, in some examples the map (1.1) is known to be nonzero, as we now explain.

Suppose we consider a theory of class $S$ obtained by compactifying the 6 d $(2,0)$ theory on a surface $C$. In this case one can construct a surface defect by starting with a surface defect of the $(2,0)$ theory and placing it at a point $z \in C$; these defects were introduced and studied in $[1,2]$. For this surface defect we have (up to discrete covers)

$$
\mathcal{M}_{2 \mathrm{~d}}=C \text {. }
$$

On the other hand, one of the essential insights of [3] was that (up to discrete covers) $\mathcal{M}_{4 \mathrm{~d}}$ is the Teichmüller space of $C$. It follows that

$$
T \mathcal{M}_{4 \mathrm{~d}}=\operatorname{Def}(C) .
$$

Thus in this case we actually have

$$
T \mathcal{M}_{4 \mathrm{~d}}=\operatorname{Def}\left(\mathcal{M}_{2 \mathrm{~d}}\right)
$$

in other words, the map (1.1) is not only nonzero but an isomorphism.

Computing the deformation intrinsically. The question we address in this paper is: how can the map (1.1) be understood and calculated intrinsically in the language of QFT, without relying on class $S$ descriptions or other features of specific examples?

Here is the answer we propose. Let $R_{4 \mathrm{~d}}$ denote the space of chiral operators of dimension 2 in the bulk $\mathcal{N}=2$ theory. Any operator $\Phi \in R_{4 \mathrm{~d}}$ has a descendant $Q^{4} \Phi$ which can 
be used to deform the theory in a way which preserves $d=4, \mathcal{N}=2$ supersymmetry and conformal invariance; this gives an identification

$$
R_{4 \mathrm{~d}}=T \mathcal{M}_{4 \mathrm{~d}} .
$$

Likewise, let $R_{2 \mathrm{~d}}$ denote the space of chiral local operators of dimension 1 living on the surface defect. Any operator $\Sigma \in R_{2 \mathrm{~d}}$ can be used to deform the surface defect in a way which preserves $d=2, \mathcal{N}=(2,2)$ supersymmetry and conformal invariance; this gives an identification

$$
R_{2 \mathrm{~d}}=T \mathcal{M}_{2 \mathrm{~d}}
$$

Now suppose given $\Phi \in R_{4 \mathrm{~d}}$ and $\bar{\Sigma} \in \overline{R_{2 \mathrm{~d}}}$. We let $\mu_{\Phi}(\bar{\Sigma})$ denote the most singular term in the bulk-defect OPE,

$$
\Phi(x) \bar{\Sigma}(0)=\frac{4}{\mathrm{i} \pi} \frac{\mu_{\Phi}(\bar{\Sigma})}{|x|^{2}}+\cdots
$$

Counting R-charges and dimensions shows that $\mu_{\Phi}(\bar{\Sigma}) \in R_{2 \mathrm{~d}}$. Thus we have obtained a linear map

$$
\mu_{\Phi}: \overline{R_{2 \mathrm{~d}}} \rightarrow R_{2 \mathrm{~d}}
$$

Since $R_{2 \mathrm{~d}}=T \mathcal{M}_{2 \mathrm{~d}}$, such a map can be interpreted as

$$
\mu_{\Phi}: \overline{T \mathcal{M}_{2 \mathrm{~d}}} \rightarrow T \mathcal{M}_{2 \mathrm{~d}},
$$

or equivalently $\mu_{\Phi} \in \Omega^{0,1}\left(\mathcal{M}_{2 \mathrm{~d}}, T \mathcal{M}_{2 \mathrm{~d}}\right)$. Moreover, $\mu_{\Phi}$ obeys the condition $\bar{\partial} \mu_{\Phi}=0$. As we review in appendix $\mathrm{C}$, the $\bar{\partial}$-cohomology class of such a $\mu_{\Phi}$ determines an element

$$
\left[\mu_{\Phi}\right] \in \operatorname{Def}\left(\mathcal{M}_{2 \mathrm{~d}}\right) .
$$

Our main claim is that $\left[\mu_{\Phi}\right]$ represents the deformation of $\mathcal{M}_{2 \mathrm{~d}}$ which is induced by perturbing the bulk theory using the operator $Q^{4} \Phi$.

Our derivation of this claim is given in section 3 below. It builds on the study of operator mixing in deformations of CFTs [4-7]. It was argued in these works that as a CFT is deformed, the OPE between the perturbing operator and other local operators determines a connection on the vector bundle of local operators. This connection is responsible for the phenomenon of mixing between local operators as we move on the conformal manifold of the CFT. In a similar way, we find that the OPE between local operators in the bulk QFT and local operators inserted on the defect contains information about operator mixing between marginal chiral descendants $Q^{2} \Sigma$ and marginal anti-chiral descendants $\bar{Q}^{2} \bar{\Sigma}$ as we move on the moduli space $\mathcal{M}_{4 \mathrm{~d}}$. This operator mixing is a manifestation of the deformation of complex structure of $\mathcal{M}_{2 \mathrm{~d}}$.

In section 4 we discuss one concrete example, where the bulk theory is the pure $\mathcal{N}=2$ theory with gauge group U(1), and the defect is a supersymmetric "solenoid." In this case the moduli space $\mathcal{M}_{2 \mathrm{~d}}$ is a 1-dimensional complex torus, whose complex modulus is the $4 \mathrm{~d}$ gauge coupling $\tau$. We verify in this case that the OPE between the bulk deformation operator $\Phi=\phi^{2}$ and the defect anti-chiral operator $\bar{\Sigma}=\bar{\phi}$ gives the Beltrami differential associated to the complex deformation of $\mathcal{M}_{2 \mathrm{~d}}$ as expected. (We remark that this example can be thought of as a class $S$ theory associated to the Lie algebra $g l(1)$ where $C$ is a torus.) 


\section{Comments and future directions:}

1. In this paper we examine one very specific deformation problem, that of moduli spaces of $\frac{1}{2}$-BPS surface defects in $4 \mathrm{~d} \mathcal{N}=2$ theories. The basic mechanism we find, that these deformations are controlled by the bulk-defect OPE, seems likely to recur in other dimensions and other amounts of supersymmetry. It would be interesting to explore other examples.

2. Although our analysis is intended to apply to a general $\mathcal{N}=2$ theory and surface defect, the only example we consider in detail is that of a free theory and surface defect. It would be interesting to verify our analysis directly in an interacting theory. For example, we could consider the pure SU(2) theory with $N_{f}=4$. This theory has a class $S$ realization where $C$ is a four-punctured sphere, and a corresponding canonical surface defect with $\mathcal{M}_{2 \mathrm{~d}}=C$; if we consider the bulk-defect OPE in this theory we thus expect to obtain a Beltrami differential representing the deformation of the four-punctured sphere which changes the cross-ratio of the punctures.

3. One of the technical tools in our analysis is a computation of part of the covariant derivative of the conserved supercurrent of the $4 \mathrm{~d} \mathcal{N}=2$ theory with respect to the coupling: we find that (at least with the regularization scheme we use) the supercurrent $\bar{J}$ mixes with a descendant of the chiral perturbation $\Phi$, schematically $\nabla_{\epsilon} \bar{J}=Q^{3} \Phi+\cdots$ (see section 3.3 for the precise statement). This kind of mixing might occur more generally for deformations of supersymmetric theories, and if so it could be interesting to study more systematically.

4. In this paper we focus on marginal deformations of the bulk theory, descending from chiral operators $\Phi$ of dimension 2. One could similarly consider $\Phi$ of dimension $2+k$ with $k>0$, which would give rise to irrelevant deformations of the bulk theory. The bulk-defect OPE then gives a map from defect anti-chiral operators of dimension 1 to defect chiral operators of dimension $1+k$. When $C$ is 1-dimensional, such a map can be interpreted as a higher Beltrami differential on $C$, and thus irrelevant perturbations of the bulk theory correspond to perturbations of a higher complex structure on $C$ in the sense of [8]. It would be very interesting to understand the meaning of this higher complex structure on $C$ in terms of the physics of the surface defect; one intriguing possibility is to interpret it as a deformation of a larger moduli space including both marginal and irrelevant deformations of the defect.

\section{Acknowledgments}

We thank Chris Beem, David Ben-Zvi, Jacques Distler, Aaron Fenyes, Greg Moore and Kyriakos Papadodimas for helpful conversations. The work of AN was supported by NSF grants DMS-2005312 and DMS-1711692. AN also thanks the Mathematical Sciences Research Institute for hospitality during the fall 2019 semester, supported by NSF grant DMS-1440140. The work of AS was supported by NSF grant DMS-1711692. 


\begin{tabular}{|c|c|c|c|c|c|c|c|c|}
\hline 4d notation & $Q^{1}{ }_{1}$ & $Q^{2}{ }_{2}$ & $\bar{Q}_{1 \mathrm{i}}$ & $\bar{Q}_{2 \dot{2}}$ & $S_{1}{ }^{1}$ & $S_{2}{ }^{2}$ & $\bar{S}^{1 \mathrm{i}}$ & $\bar{S}^{2 \dot{2}}$ \\
\hline 2d notation & $G_{-1 / 2}^{+}$ & $\bar{G}_{-1 / 2}^{-}$ & $G_{-1 / 2}^{-}$ & $\bar{G}_{-1 / 2}^{+}$ & $G_{1 / 2}^{-}$ & $\bar{G}_{1 / 2}^{+}$ & $G_{1 / 2}^{+}$ & $\bar{G}_{+1 / 2}^{-}$ \\
\hline $\mathfrak{u}(1)_{V}$ & +1 & +1 & -1 & -1 & -1 & -1 & +1 & +1 \\
\hline $\mathfrak{u}(1)_{A}$ & +1 & -1 & -1 & +1 & -1 & +1 & +1 & -1 \\
\hline $\mathfrak{s o}(2)_{34}$ & $+\frac{1}{2}$ & $-\frac{1}{2}$ & $+\frac{1}{2}$ & $-\frac{1}{2}$ & $-\frac{1}{2}$ & $+\frac{1}{2}$ & $-\frac{1}{2}$ & $+\frac{1}{2}$ \\
\hline
\end{tabular}

Table 1. The supercharges preserved in the presence of a surface defect and their charges under $\mathfrak{u}(1)_{V}, \mathfrak{u}(1)_{A}$ and $\mathfrak{s o}(2)_{34}$. The normalization of $2 \mathrm{~d}$ operators in terms of the $4 \mathrm{~d}$ notation contains factors of $\frac{1}{2}$ (see equations (B.9), (B.10).)

\section{Properties of $2 \mathrm{~d}-4 \mathrm{~d}$ systems}

This section is divided as follows. In section 2.1, we review the superconformal algebra of 2d-4d systems. Explicit commutation relations are not given here but can be found in appendix A and appendix B. In section 2.2 we discuss supersymmetry-preserving marginal operators of $4 \mathrm{~d}$ and $2 \mathrm{~d}$ systems.

\subsection{Superconformal symmetry of $2 d-4 d$ systems}

We start by reviewing the $\mathcal{N}=2$ superconformal algebra in four dimensions. Its bosonic subalgebra is $\mathfrak{s o}(5,1) \oplus \mathfrak{s u}(2)_{r} \oplus \mathfrak{u}(1)_{r}$ and the fermionic generators transform in a doublet of $\mathfrak{s u}(2)_{r}$ as well as a spinor representation of $\mathfrak{s o}(5,1)$. The $\mathfrak{u}(1)_{r}$ charges of these generators are given in the paragraph below. Under the decomposition $\mathfrak{s o}(4) \oplus \mathfrak{s o}(1,1) \subset \mathfrak{s o}(5,1)$, a Weyl representation of $\mathfrak{s o}(5,1)$ decomposes into Weyl representations of $\mathfrak{s o}(4)$ distinguished by the action of $\mathfrak{s o}(1,1)$. The generators with eigenvalues $+\frac{1}{2}$ and $-\frac{1}{2}$ are called Poincaré and conformal supercharges respectively.

Using the isomorphism $\mathfrak{s o}(4) \cong \mathfrak{s u}(2)_{L} \oplus \mathfrak{s u}(2)_{R}$, we denote the Poincaré supercharges as $Q^{i}{ }_{\alpha}, \bar{Q}_{j \dot{\alpha}}$ and the conformal supercharges as $S_{i}{ }^{\alpha}$ and $\bar{S}^{i \dot{\alpha}}$. Here $\alpha, \dot{\alpha}$ and $i$ index components in the fundamental representations of $\mathfrak{s u}(2)_{L}, \mathfrak{s u}(2)_{R}$ and $\mathfrak{s u}(2)_{r}$ respectively. Under the action of $\mathfrak{u}(1)_{R}, Q$ 's and $\bar{S}$ 's carry charge +1 while $S$ 's and $\bar{Q}$ 's carry charge -1 .

In the presence of a surface defect $\mathrm{S}$ on a plane $P$, the algebra of conformal symmetries $\mathfrak{s o}(5,1)$ is reduced to $\mathfrak{s o}(3,1) \oplus \mathfrak{s o}(2)_{\perp}$. Here $\mathfrak{s o}(2)_{\perp}$ is the algebra of rotation symmetry in the plane orthogonal to $P$. If $P$ spans $\mathbb{R}_{x^{1}=x^{2}=0}^{2}$, then, with the conventions listed in appendix A, the list of fermionic symmetries is given in table 1 .

The unbroken symmetries generate the two-dimensional $(2,2)$ superconformal algebra as is evident by studying the action of unbroken bosonic symmetries on the supercharges. The $\mathfrak{u}(1)_{r}$ symmetry of $\mathcal{N}=2$ algebra is preserved; its charge is identified with half the charge of $\mathfrak{u}(1)_{V}$ R-symmetry. The $\mathfrak{s u}(2)_{R}$ symmetry is explicitly broken to its Cartan, generated in our notation by $R_{3}$. The sub-algebra of $\mathfrak{s u}(2)_{R} \oplus \mathfrak{s o}(2)_{\perp}$ generated by $4 R_{3}+2 J_{12}$ is naturally identified with the axial R-symmetry in two dimensions. Finally, the $\mathfrak{u}(1)$ algebra generated by $J_{12}+R_{3}$ is the commutant of the embedding. 


\subsection{Descendants of superconformal primaries}

In this subsection, we discuss certain supersymmetry-preserving marginal operators of $4 \mathrm{~d}$ $\mathcal{N}=2$ and $2 \mathrm{~d} \mathcal{N}=(2,2)$ systems. The operators that we discuss are distinguished by the properties that they are supersymmetric descendants of primary operators, and that they are marginal.

Descendants of primaries in $4 \mathbf{d} \mathcal{N}=\mathbf{2}$ systems. A superconformal primary is a local operator annihilated by the conformal supercharges $S_{i}{ }^{\alpha}$ and $\bar{S}^{i \dot{\alpha}}$. If this operator is further annihilated by the right-handed (left-handed) Poincare supercharges it is called a chiral (anti-chiral) primary. If we assume that such an operator is a Lorentz scalar, its scaling dimension and $\mathfrak{u}(1)_{r}$ charge satisfy the equality

$$
\Delta=\mp r
$$

where the upper (lower) sign holds for chiral (anti-chiral) primaries.

We obtain supersymmetry-preserving marginal operators through the action of lefthanded (right-handed) Poincaré supercharges on chiral (anti-chiral) primaries of scaling dimension $\Delta=2$. We denote these primaries as $\Phi_{k}\left(\bar{\Phi}_{k}\right)$; and the marginal operators as $Q^{4} \Phi_{k}\left(\bar{Q}^{4} \bar{\Phi}_{k}\right)$.

$$
\begin{aligned}
Q^{4} \Phi_{k} & :=\frac{\mathrm{i}}{64 \pi} Q^{1}{ }_{1} Q^{1}{ }_{2} Q^{2}{ }_{1} Q^{2}{ }_{2} \Phi_{k} \\
\bar{Q}^{4} \bar{\Phi}_{k} & :=-\frac{\mathrm{i}}{64 \pi} \bar{Q}_{1 \mathrm{i}} \bar{Q}_{12} \bar{Q}_{2 \mathrm{i}} \bar{Q}_{2 \dot{2}} \bar{\Phi}_{k}
\end{aligned}
$$

Here $k$ goes from 1 to $N$ - the number of such operators in a theory. $Q^{4} \Phi_{k}$ and $\bar{Q}^{4} \bar{\Phi}_{k}$ are complex conjugates in a spacetime with Minkowski signature; however, they are not complex conjugate in the Euclidean signature. This is because the Weyl spinors of $\mathfrak{s o}(3,1)$ are complex conjugate while those of $\mathfrak{s o}(4)$ are pseudo-real. In this paper, we will work in $\mathbb{R}^{4}$.

Descendants of primaries in $2 \mathrm{~d} \mathcal{N}=(2,2)$ systems. In the notation of $2 \mathrm{~d} \mathcal{N}=$ $(2,2)$ algebra the supercharges are denoted by $G_{r}^{a}$ and $\bar{G}_{r}^{a}$ where $a \in\{ \pm\}$ and $r \in\left\{ \pm \frac{1}{2}\right\}$ (see appendix B). The generators with $r=-\frac{1}{2}$ are the Poincaré supercharges while those with $r=+\frac{1}{2}$ are the conformal supercharges. Similar to the case of $4 \mathrm{~d} \mathcal{N}=2$, a superconformal primary is defined as an operator that is annihilated by all of the conformal supercharges. As is well known (and is reviewed in appendix B), the algebra of $2 \mathrm{~d} \mathcal{N}=(2,2)$ supersymmetry splits into a holomorphic and an anti-holomorphic sector. Hence the discussion of chiral and anti-chiral primaries also splits.

In the holomorphic sector, the operators annihilated by $G_{-1 / 2}^{+}$are called chiral primaries, while the operators annihilated by $G_{-1 / 2}^{-}$are called anti-chiral primaries. The $L_{0}$ and $J_{0}$ eigenvalues of these operators satisfy

$$
l_{0}= \pm \frac{j_{0}}{2}
$$


where the upper (lower) sign holds for chiral (anti-chiral) primaries. After taking into account the anti-holomorphic sector, the full $\mathcal{N}=(2,2)$ algebra admits four kinds of primary operators labelled as $(a, a),(a, c),(c, a)$ and $(c, c)$. We will also refer to these operators as twisted chiral, chiral, anti-chiral and anti-twisted chiral operators respectively.

The supersymmetry preserving marginal operators are descendants of the primary operators of $\left(l_{0}, \bar{l}_{0}\right)$ weights equal to $\left(\frac{1}{2}, \frac{1}{2}\right)$, obtained by the action of those supercharges that do not annihilate them. For example, the descendant of a chiral primary $\Sigma(x)$ is

$$
Q^{2} \Sigma=\frac{1}{2 \sqrt{2}} Q^{1}{ }_{1} Q_{2}^{2} \Sigma=\sqrt{2} G_{-1 / 2}^{+} \bar{G}_{-1 / 2}^{-} \Sigma
$$

Similarly, the descendant of an anti-chiral primary $\bar{\Sigma}$ is

$$
\bar{Q}^{2} \bar{\Sigma}=-\frac{1}{2 \sqrt{2}} \bar{Q}_{1 i} \bar{Q}_{2 \dot{2}} \bar{\Sigma}=-\sqrt{2} G_{-1 / 2}^{-} \bar{G}_{-1 / 2}^{+} \bar{\Sigma}
$$

\section{Deformations of $4 \mathrm{~d}$ and $2 \mathrm{~d}-4 \mathrm{~d}$ systems}

\subsection{Deformation of pure $4 d$ systems}

Consider deforming a $4 \mathrm{~d} \mathcal{N}=2$ theory by a marginal chiral descendant,

$$
\delta S=\epsilon \int_{\mathbb{R}^{4}} \Phi^{(4)},
$$

where we defined

$$
\Phi^{(4)}(x):=Q^{4} \Phi(x) \mathrm{d}^{4} x ;
$$

the superscript in $\Phi^{(4)}$ denotes that this object is a 4 -form. In this section we briefly review the effect of this deformation on the correlation functions. We use the notation that $\langle\cdots\rangle_{\epsilon}$ is the deformed correlation function while $\langle\cdots\rangle_{0}$ is the undeformed correlation function.

Formal path-integral manipulations would say that the first derivative of $\langle\cdots\rangle_{\epsilon}$ with respect to $\epsilon$ is

$$
\left.\frac{\mathrm{d}}{\mathrm{d} \epsilon}\left\langle\prod_{i=1}^{n} \mathcal{O}_{i}\left(x_{i}\right)\right\rangle_{\epsilon}\right|_{\epsilon=0}=-\int_{\mathbb{R}^{4}}\left\langle\Phi^{(4)}(x) \prod_{i=1}^{n} \mathcal{O}_{i}\left(x_{i}\right)\right\rangle_{0}
$$

As it is written, the right side of (3.3) is potentially ill-defined, as there may be a shortdistance singularity as $x \rightarrow x_{i}$. In order to cure this problem, one can follow a $4 \mathrm{~d}$ version of a regularization procedure discussed e.g. in [7] in the $2 \mathrm{~d}$ context. ${ }^{1}$ That procedure entails cutting out small balls around the points of insertion of local operators, computing the integrated correlation function as a function of the radii of these balls, and dropping divergent terms as the radii are taken to zero. ${ }^{2}$

With this sort of regularization understood, (3.3) computes the correlation functions of a family of $4 \mathrm{~d} \mathcal{N}=2$ theories, to first order around a point of $\mathcal{M}_{4 \mathrm{~d}}$. By construction

\footnotetext{
${ }^{1}$ In particular, the regularization procedure discussed here corresponds to the $\bar{c}$-connection in [7].

${ }^{2} \mathrm{An}$ alternative approach to regularization is through the addition of contact terms in the OPE of the operators whose points of insertion coincide. This method is discussed in [4,5], and as explained in footnote 10 of [9], is equivalent to the procedure of [7].
} 
the theories at $\epsilon=0$ and $\epsilon \neq 0$ have the same vector space of local operators, since in (3.3) the insertions in the correlation functions $\langle\cdots\rangle_{\epsilon}$ are drawn from the original space of local operators, even when $\epsilon \neq 0$.

So far, so good, to first order in $\epsilon$. When one tries to go to higher order, however, the situation becomes more complicated. Following [9], one can take some inspiration from the case of 2-dimensional field theories, discussed at some length in [7] following previous works including [4-6] (see also [10]). The results of [9] have been further developed and applied to various aspects of $4 \mathrm{~d} \mathcal{N}=2$ theories, e.g. [11-14]. The expected picture can be summarized as follows: the space $\mathcal{M}_{4 \mathrm{~d}}$ carries a vector bundle whose fiber over a point $\tau \in \mathcal{M}_{4 \mathrm{~d}}$ is the space of local operators of the theory with coupling $\tau$, but this vector bundle is not naturally trivial. The fact noted above, that we can identify operators at $\epsilon=0$ and $\epsilon \neq 0$ to first order in $\epsilon$, means that the bundle of local operators carries a connection $\nabla$. The connection $\nabla$ depends on the regularization scheme, and may have curvature in general, which is one manifestation of the phenomenon of operator mixing under marginal perturbations.

Revisiting (3.3) from this perspective, we see that the appropriate interpretation is that this equation is true when the $\mathcal{O}_{i}$ are sections of the bundle of local operators obeying $\nabla_{\epsilon} \mathcal{O}_{i}=0$; this is the invariant way of saying the operator insertions are "independent of $\epsilon$." More generally, if the $\epsilon$ dependence of the operators $\mathcal{O}_{i}$ is chosen arbitrarily, (3.3) is replaced by

$$
\left.\frac{\mathrm{d}}{\mathrm{d} \epsilon}\left\langle\prod_{i=1}^{n} \mathcal{O}_{i}\left(x_{i}\right)\right\rangle_{\epsilon}\right|_{\epsilon=0}=-\int_{\mathbb{R}^{4}}\left\langle\Phi^{(4)}(x) \prod_{i=1}^{n} \mathcal{O}_{i}\left(x_{i}\right)\right\rangle_{0}+\sum_{j=1}^{n}\left\langle\nabla_{\epsilon} \mathcal{O}_{j}\left(x_{j}\right) \prod_{i \neq j, i=1}^{n} \mathcal{O}_{i}\left(x_{i}\right)\right\rangle_{0}
$$

\subsection{Deformations of $2 \mathrm{~d}-4 \mathrm{~d}$ systems}

Now we consider adding a $\frac{1}{2}$-BPS surface defect to the system and deforming again by a bulk $4 \mathrm{~d}$ chiral descendant. We assume that the surface defect continues to be $\frac{1}{2}$-BPS in the deformed theory.

Working formally, as above, deformed correlation functions to first order can be computed by the analogue of (3.3),

$$
\left.\frac{\mathrm{d}}{\mathrm{d} \epsilon}\left\langle\mathrm{S}(P) \prod_{i=1}^{n} \mathcal{O}_{i}^{\mathrm{b}}\left(x_{i}\right) \prod_{i=1}^{k} \mathcal{O}_{i}^{\mathrm{d}}\left(y_{i}\right)\right\rangle_{\epsilon}\right|_{\epsilon=0}=-\int_{\mathbb{R}^{4}}\left\langle\Phi^{(4)}(x) \mathrm{S}(P) \prod_{i=1}^{n} \mathcal{O}_{i}^{\mathrm{b}}\left(x_{i}\right) \prod_{i=1}^{k} \mathcal{O}_{i}^{\mathrm{d}}\left(y_{i}\right)\right\rangle_{0},
$$

where $\mathrm{S}(P)$ denotes the surface defect inserted on the plane $P$, bulk local operators are inserted at points $x_{i} \in \mathbb{R}^{4}$, defect local operators at points $y_{i} \in P$. The equation (3.5) needs to be understood as including a regularization, e.g. cutting out a tubular neighborhood of the defect and taking its radius to zero with divergent terms dropped, in parallel to what we discussed for the bulk local operators. ${ }^{3}$

\footnotetext{
${ }^{3}$ One might have imagined a more general situation, namely that the deformed correlation functions given by (3.5) are not those of an $\mathcal{N}=(2,2)$ supersymmetric theory, but they become supersymmetric after shifting the action on the surface defect by a marginal operator. However, all of the marginal operators in an $\mathcal{N}=(2,2)$ superconformal theory actually correspond to supersymmetry-preserving deformations. Thus, if the theory is supersymmetric with such an addition, it is also supersymmetric without one; this means we can restrict our attention to the case where we do not make such an addition.
} 
To go beyond first-order deformations, as before, one needs to be more careful. While we have not developed the theory in a fully systematic way, we propose the following picture, parallel to the picture for $4 \mathrm{~d}$ local operators which we reviewed above.

First, given a regularization scheme as we sketched above, we should obtain an Ehresmann connection in the fiber bundle $\mathcal{M}_{2 \mathrm{~d}-4 \mathrm{~d}} \rightarrow \mathcal{M}_{4 \mathrm{~d}}$; this Ehresmann connection defines what it means for the surface defect to be "independent of $\epsilon$." As with the connection on local operators, this Ehresmann connection in $\mathcal{M}_{2 \mathrm{~d}-4 \mathrm{~d}}$ may have curvature, in the sense that homotopic paths on $\mathcal{M}_{4 \mathrm{~d}}$ may lift to different paths on $\mathcal{M}_{2 \mathrm{~d}-4 \mathrm{~d}}$. We will not need to consider that curvature here.

Second, there is a vector bundle over $\mathcal{M}_{2 \mathrm{~d}-4 \mathrm{~d}}$ consisting of defect local operators. This vector bundle should carry a connection, which defines what it means for defect local operators to be "independent of $\epsilon$." To describe this connection concretely we may use the Ehresmann connection to make a splitting between the horizontal and vertical directions in the fiber bundle $\mathcal{M}_{2 \mathrm{~d}-4 \mathrm{~d}}$. Along the horizontal lifts of tangent vectors from the base $\mathcal{M}_{4 \mathrm{~d}}$ to $\mathcal{M}_{2 \mathrm{~d}-4 \mathrm{~d}}$, the connection is determined by the regularized integrals (3.5), which give us the first-order identification between spaces of local operators on the defect. In this paper we will not need to discuss the connection in the vertical directions, but we remark that it is determined by the same procedure we used in section 3.1, now applied to a marginal perturbation of a $2 \mathrm{~d}$ theory instead of a $4 \mathrm{~d}$ theory.

We assume that the regularization scheme can be set up in such a way that $2 \mathrm{~d}$ and $4 \mathrm{~d}$ perturbations commute with one another. As a practical matter this means the following. As we have already discussed, there is a connection in the bundle of local operators over $\mathcal{M}_{2 \mathrm{~d}-4 \mathrm{~d}}$. Because the vertical tangent bundle to $\mathcal{M}_{2 \mathrm{~d}-4 \mathrm{~d}}$ is identified with the space of marginal operators on the defect, this gives a connection in the vertical tangent bundle. On the other hand, the Ehresmann connection allows us to identify nearby fibers and thus also induces a connection in the vertical tangent bundle. Our assumption is that these two connections are equal.

In parallel to the pure $4 \mathrm{~d}$ case above, we interpret (3.5) as giving the variation of the correlation functions when all of the operators $\mathrm{S}(P), \mathcal{O}_{i}^{\mathrm{b}}\left(x_{i}\right), \mathcal{O}_{i}^{\mathrm{d}}\left(y_{i}\right)$ are deformed in a covariantly constant fashion. In other words, the bulk local operators $\mathcal{O}_{i}^{\mathrm{b}}$ are covariantly constant for the connection on bulk local operators over $\mathcal{M}_{4 \mathrm{~d}}, \mathrm{~S}(P)$ is deformed along the horizontal lift of a path from $\mathcal{M}_{4 \mathrm{~d}}$ to $\mathcal{M}_{2 \mathrm{~d}-4 \mathrm{~d}}$, and the defect local operators $\mathcal{O}_{i}^{\mathrm{d}}$ are covariantly constant for the connection on defect local operators over $\mathcal{M}_{2 \mathrm{~d}-4 \mathrm{~d}}$, evaluated on the lifted path.

\subsection{Deformations of the supercurrent}

In a $4 \mathrm{~d} \mathcal{N}=2$ theory the supersymmetry transformations $Q_{\alpha}^{I}$ and $\bar{Q}_{I \dot{\alpha}}$ are generated by corresponding conserved supercurrents $J_{\alpha}^{I}$ and $\bar{J}_{I \dot{\alpha}}$ (we suppress the vector index on the currents). We argue in section $\mathrm{D}$ below that the variation of the supercurrent is given by ${ }^{4}$

$$
\nabla_{\epsilon} \bar{J}_{I \dot{\alpha}}=\left(\Phi^{(3)}\right)_{I \dot{\alpha}}^{\mu}+c \bar{J}_{I \dot{\alpha}}
$$

\footnotetext{
${ }^{4}$ Here and below, the covariant derivative $\nabla_{\epsilon}$ is understood to be computed at the point of the undeformed theory, i.e. $\epsilon=0$.
} 
where $\Phi \in R_{4 \mathrm{~d}}$ denotes the chiral operator corresponding to the coupling $\epsilon,\left(\Phi^{(3)}\right)_{I \dot{\alpha}}^{\mu}$ is the unique operator built by acting with three $Q$ 's on $\Phi$ which obeys

$$
\partial_{\mu}\left(\Phi^{(3)}\right)_{I \dot{\alpha}}^{\mu}=\bar{Q}_{I \dot{\alpha}} Q^{4} \Phi
$$

and $c$ is an undetermined constant. ${ }^{5}$

In [9] it is pointed out that the supercurrents form a bundle over $\mathcal{M}_{4 \mathrm{~d}}$ with a natural non-flat connection, which plays an important role in a four-dimensional version of $t t^{*}$ geometry. That connection should be identified with the projection of $\nabla$ onto the bundle of supercurrents, which has the effect of throwing away the $\Phi^{(3)}$ term above. In contrast, for our purposes in the rest of this paper, the $\Phi^{(3)}$ term will be the crucial part.

Below we will need to apply (3.6) in the situation where a $\frac{1}{2}$-BPS surface defect is inserted and $\bar{J}_{I \dot{\alpha}}$ is one of the supercurrents which is conserved in the presence of the defect. In this case one can worry that (3.6) might need to be corrected to include a deltafunction variation supported along the defect. In section D.3 below we argue that this is not the case: (3.6) continues to hold even in the presence of the surface defect.

\subsection{Mixing between chiral and anti-chiral descendants}

We are now ready for our main computation. We consider the connection $\nabla$ acting on defect local operators. This connection restricts to a connection on the bundle of marginal operators, and thus induces a connection on vertical tangent vectors to $\mathcal{M}_{2 \mathrm{~d}-4 \mathrm{~d}}$. In turn, the bundle of vertical tangent vectors is decomposed into holomorphic and antiholomorphic subbundles, corresponding to chiral descendants $Q^{2} \Sigma$ and anti-chiral descendants $\bar{Q}^{2} \bar{\Sigma}$ respectively. What we will compute now is the mixing between the holomorphic and antiholomorphic subbundles as we move along $\mathcal{M}_{4 \mathrm{~d}}$.

To be precise, let $\bar{\Sigma}$ denote an anti-twisted-chiral primary operator on the surface defect. We consider a first-order deformation of the bulk theory by a chiral primary $\Phi$, lift it to a first-order path in $\mathcal{M}_{2 \mathrm{~d}-4 \mathrm{~d}}$ as we have been discussing above, and extend $\bar{\Sigma}$ to a section of the bundle of anti-twisted-chiral primaries over this path. We also consider sections $J, \bar{J}$ of the bundle of conserved supercurrents over the path, and use them to define the action of the supercharges. Then the main statement of this section is that, modulo operators which are $\bar{Q}$-exact or $\bar{Q}^{\prime}$-exact, we have

$$
\nabla_{\epsilon}\left(\bar{Q}^{2} \bar{\Sigma}\right)=Q^{2}\left(\mu_{\Phi}(\bar{\Sigma})\right)
$$

with $\mu_{\Phi}$ denoting the bulk-defect OPE we defined in (1.7).

The rest of this section is taken up with the derivation of (3.8), as follows. We simplify notation a little by setting $\bar{Q}:=\bar{Q}_{2 \dot{2}}, \bar{Q}^{\prime}:=\frac{1}{2 \sqrt{2}} \bar{Q}_{11}$, so that $\bar{Q}^{2}=\overline{Q Q}^{\prime}$. Now observe that modulo $\bar{Q}$-exact terms we have

$$
\nabla_{\epsilon}\left(\bar{Q}^{2} \bar{\Sigma}\right)=\nabla_{\epsilon}\left(\overline{Q Q}^{\prime} \bar{\Sigma}\right)=\nabla_{\epsilon}(\bar{Q}) \bar{Q}^{\prime} \bar{\Sigma}
$$

\footnotetext{
${ }^{5}$ This constant cannot be determined even in principle; the reason is that by $\bar{J}$ we mean a section of the bundle of conserved supercurrents, and such a section is not quite unique; rather, it is determined up to $\bar{J} \rightarrow \mathrm{e}^{f(\epsilon)} \bar{J}$, which would change $c \rightarrow c+\partial_{\epsilon} f$.
} 
and $\nabla_{\epsilon}(\bar{Q})$ is determined by the variation of the supercurrent, given in (3.6). Continuing to work modulo $\bar{Q}$-exact terms, this allows us to rewrite the r.h.s. of (3.9) as

$$
\oint_{\Gamma} \Phi_{2 \dot{2}}^{(3)}(x) \bar{Q}^{\prime} \bar{\Sigma}(0)
$$

where $\Gamma$ denotes any 3 -cycle surrounding the point $x=0$ where the operator $\bar{\Sigma}$ was inserted, and we have used the volume element of $\mathbb{R}^{4}$ to convert $\Phi_{2 \dot{2}}^{(3)}$ from a vector field to a 3 -form (we will switch back and forth without further comment). Modulo $\bar{Q}^{\prime}$-exact terms this is equal to

$$
\oint_{\Gamma} \bar{Q}^{\prime} \Phi_{2 \dot{2}}^{(3)}(x) \bar{\Sigma}(0) .
$$

All that remains is to evaluate this integral, for which we can choose any convenient 3-cycle $\Gamma$. Let $(\rho, \psi)$ denote polar coordinates in the plane $P$ where the surface defect lies, and $(r, \theta)$ denote polar coordinates in the orthogonal plane. We choose $\Gamma$ to be the boundary of the locus $|r| \leq R,|\rho| \leq D$ for some $R, D>0$. Thus $\Gamma=\mathcal{R} \cup \mathcal{R}^{\prime}$ where

$$
\mathcal{R}=\{|r|=R,|\rho| \leq D\}, \quad \mathcal{R}^{\prime}=\{|r| \leq R,|\rho|=D\} .
$$

In the limit $R \rightarrow 0$, the contribution from $\mathcal{R}^{\prime}$ vanishes. ${ }^{6}$ Thus we can get the correct answer by computing the integral only over $\mathcal{R}$ for any fixed $D$ and then taking $R \rightarrow 0$.

Since the only bracket $\left\{\bar{Q}_{2 \dot{2}}, \cdot\right\}$ containing $P_{r}$ is $\left\{\bar{Q}_{2 \dot{2}}, Q^{2}{ }_{1}\right\}=2 \mathrm{e}^{-\mathrm{i} \theta}\left(P_{r}-\frac{\mathrm{i}}{r} P_{\theta}\right)$, using (2.2) and (3.7) gives

$$
\Phi_{r}^{(3)}=-\frac{1}{32 \pi} \mathrm{e}^{-\mathrm{i} \theta} Q^{1}{ }_{1} Q^{1}{ }_{2} Q^{2}{ }_{2} \Phi .
$$

Furthermore, the operator $\bar{Q}^{\prime} \Phi_{r}^{(3)}$ is a total derivative $\partial_{\mu} \mathcal{X}^{\mu}$, because the anticommutator of $\bar{Q}^{\prime}=\frac{\bar{Q}_{11}}{2 \sqrt{2}}$ with $Q^{1}{ }_{1}$ and $Q^{1}{ }_{2}$ gives translations. Thus we have

$$
\oint_{\mathcal{R}} \mathrm{d}^{3} x \bar{Q}^{\prime} \Phi_{r}^{(3)}(x) \bar{\Sigma}(0)=\oint_{\mathcal{R}} \mathrm{d}^{3} x \partial_{\mu} \mathcal{X}^{\mu}(x) \bar{\Sigma}(0) .
$$

We use the decomposition $\partial_{\mu} \mathcal{X}^{\mu}=\partial_{\theta} \mathcal{X}^{\theta}+\partial_{\psi} \mathcal{X}^{\psi}+\partial_{r} \mathcal{X}^{r}+\partial_{\rho} \mathcal{X}^{\rho}$, and consider the four terms in turn:

- The terms involving $\partial_{\psi} \mathcal{X}^{\psi}$ and $\partial_{\theta} \mathcal{X}^{\theta}$ vanish since they are integrals of total derivatives over the circle.

- The term with $\partial_{\rho} \mathcal{X}^{\rho}$ reduces to the boundary integral $\oint_{S_{R}^{1} \times S_{D}^{1}} \mathcal{X}^{\rho}$. In the limit $R \rightarrow 0$, this boundary integral can be nonzero only if there is a singularity of order $\frac{1}{r}$ in the OPE between $\chi^{\rho}$ and the surface defect; in the next paragraph we show there is no such singularity.

Since the only bracket $\left\{\bar{Q}_{1 \mathrm{i}}, \cdot\right\}$ containing $P_{\rho}$ is $\left\{\bar{Q}_{1 \mathrm{i}}, Q^{1}{ }_{1}\right\}=-2 \mathrm{e}^{-\mathrm{i} \psi}\left(P_{\rho}+\frac{\mathrm{i}}{r} P_{\psi}\right)$ we have

$$
\chi^{\rho}=\frac{1}{32 \sqrt{2} \pi} \mathrm{e}^{-\mathrm{i} \psi-\mathrm{i} \theta} Q_{2}^{1} Q_{2}^{2} \Phi .
$$

\footnotetext{
${ }^{6}$ Note that this integral is convergent despite the presence of the surface defect at $r=0$; indeed the worst possible singularity in the OPE between $\Phi_{2 \dot{2}}^{(3)}$ and the surface defect is of order $1 / r$, as discussed in section D.3, and this singularity is integrable.
} 
Now we consider the OPE of $Q^{1}{ }_{2} Q^{2}{ }_{2} \Phi$ with the surface defect. As is discussed in section D.3, the most singular term in this expansion has the form $\frac{\mathrm{e}^{\mathrm{i} \theta}}{r} Q^{2}{ }_{2} \xi$, where $\xi$ is a superconformal primary operator on the defect obeying $Q^{1}{ }_{1} Q^{2}{ }_{2} \xi=0$; it follows that $Q_{2}^{2} \xi=0$, so the $\frac{1}{r}$ term vanishes, as desired.

- Thus the only term that remains from (3.14) is

$$
\oint_{\mathcal{R}} \mathrm{d}^{3} x \partial_{r} \mathcal{X}^{r}(x) \bar{\Sigma}(0)
$$

Since the only bracket $\left\{\bar{Q}_{1 \mathrm{i}}, \cdot\right\}$ containing $P_{r}$ is $\left\{\bar{Q}_{1 \mathrm{i}}, Q^{1}{ }_{2}\right\}=2 \mathrm{e}^{\mathrm{i} \theta}\left(P_{r}+\frac{\mathrm{i}}{r} P_{\theta}\right)$, we have

$$
\mathcal{X}^{r}=-\frac{\mathrm{i}}{32 \sqrt{2} \pi} Q^{1}{ }_{1} Q_{2}^{2} \Phi
$$

so this integral becomes

$$
\oint_{\mathcal{R}} \mathrm{d}^{3} x \partial_{r} \mathcal{X}^{r}(x) \bar{\Sigma}(0)=-\frac{\mathrm{i}}{32 \sqrt{2} \pi} Q^{1}{ }_{1} Q^{2}{ }_{2}\left(\oint_{\mathcal{R}} \mathrm{d}^{3} x \partial_{r} \Phi(x) \bar{\Sigma}(0)\right) .
$$

To go further we replace $\Phi(x) \bar{\Sigma}(0)$ by an OPE expansion involving operators inserted at 0 . The $(\Delta, r)$ quantum numbers of $\Phi$ and $\bar{\Sigma}$ are $(2,-2)$ and $(1,1)$ respectively. Therefore, the most singular term in the $\operatorname{OPE}$ has $(\Delta, r)=(1,-1)$ and is hence a chiral operator on the defect, which we denote as $\mu_{\Phi}(\bar{\Sigma})$ :

$$
\Phi(x) \bar{\Sigma}(0)=\frac{4}{\mathrm{i} \pi} \frac{\mu_{\Phi}(\bar{\Sigma})}{|x|^{2}}+\cdots
$$

Note that the only singularity in this OPE occurs at $x=0$, the point of the surface defect where $\bar{\Sigma}$ is inserted. One might have worried that there would also be a singularity when $y$ lies at a general point of the surface defect. However, such a singularity does not occur, for the following reason. Since $\Phi$ is a bulk chiral operator, its scaling dimension and $\mathfrak{u}(1)_{r}$ charge satisfy $\Delta=-r$. In a unitary theory, the bulk-to-defect OPE of $\Phi$ contains no singular terms, as the operators in such terms would have lower scaling dimension but the same $\mathfrak{u}(1)_{r}$ charge, violating the unitarity bound $\Delta \geq|r|$.

Now we are in position to calculate the integral: (3.18) becomes

$$
-\frac{\mathrm{i}}{16 \pi} \frac{4}{\mathrm{i} \pi}\left(\oint_{\mathcal{R}} \partial_{r} \frac{1}{|x|^{2}}\right) Q^{2} \mu_{\Phi}(\bar{\Sigma})
$$

and we have

$$
\oint_{\mathcal{R}} \mathrm{d}^{3} x \partial_{r} \frac{1}{r^{2}+\rho^{2}}=-4 \pi^{2} R \int_{0}^{D} \rho \mathrm{d} \rho \frac{2 R}{\left(R^{2}+\rho^{2}\right)^{2}}=-4 \pi^{2} \frac{D^{2}}{R^{2}+D^{2}}
$$

which in the limit $R \rightarrow 0$ becomes simply $-4 \pi^{2}$, independent of $D$ as expected; substituting this in (3.20) gives the final result

$$
Q^{2} \mu_{\Phi}(\bar{\Sigma})
$$

matching (3.8) as desired. 


\subsection{The complex structure deformation}

Our main result (3.8) expresses the phenomenon of mixing between holomorphic and antiholomorphic tangent vectors to $\mathcal{M}_{2 \mathrm{~d}}$ as we move along the lift of a path in $\mathcal{M}_{4 \mathrm{~d}}$. What remains is to explain how this mixing is related to the infinitesimal deformation of complex structure of $\mathcal{M}_{2 \mathrm{~d}}$.

We first consider the properties of the tensor $\mu_{\Phi} \in \Omega^{0,1}\left(\mathcal{M}_{2 \mathrm{~d}}, T \mathcal{M}_{2 \mathrm{~d}}\right)$ defined by (3.19). We have (up to irrelevant constant factors)

$$
\left\langle\Psi \mid \mu_{\Phi}(\bar{\Sigma})\right\rangle=|x|^{2}\langle\Psi \mid \Phi(x) \bar{\Sigma}(0)\rangle .
$$

where $\Psi$ is an anti-chiral operator. In components on $\mathcal{M}_{2 \mathrm{~d}}$, we could write this as

$$
\left(\mu_{\Phi}\right)_{\bar{a}}^{b} g_{b \bar{b}}=|x|^{2}\left\langle\bar{\Sigma}_{\bar{b}} \mid \Phi(x) \bar{\Sigma}_{\bar{a}}(0)\right\rangle .
$$

By placing $x$ on the defect (using the fact that there is no singularity in the bulk-defect OPE as noted above), we can regard $\Phi$ as a chiral operator of the defect theory; then making a conformal transformation we have

$$
\left(\mu_{\Phi}\right)_{\bar{a}}^{b} g_{b \bar{b}}=\left\langle\Phi(\infty) \bar{\Sigma}_{\bar{a}}(1) \bar{\Sigma}_{\bar{b}}(0)\right\rangle .
$$

This is an anti-extremal correlator on the defect, since it involves one chiral operator and several anti-chiral operators. It is a familiar fact from $t t^{*}$ geometry [15] that these correlators are covariantly antiholomorphic, and that the three-point correlator obeys an integrability condition, which in this case reads ${ }^{7}$

$$
\left(\nabla_{\bar{c}} \mu_{\Phi}\right)_{\bar{a}}^{b}=\left(\nabla_{\bar{a}} \mu_{\Phi}\right)_{\bar{c}}^{b}
$$

Thus $\mu_{\Phi} \in \Omega^{0,1}\left(\mathcal{M}_{2 \mathrm{~d}}, T \mathcal{M}_{2 \mathrm{~d}}\right)$ is antiholomorphic and $\bar{\partial}$-closed.

The fact that $\mu_{\Phi}$ is $\bar{\partial}$-closed means it defines a class

$$
\left[\mu_{\Phi}\right] \in H^{1}\left(\mathcal{M}_{2 \mathrm{~d}}, T \mathcal{M}_{2 \mathrm{~d}}\right)=\operatorname{Def}\left(\mathcal{M}_{2 \mathrm{~d}}\right)
$$

(see appendix $\mathrm{C}$ for a quick review). We claim that the class $\left[\mu_{\Phi}\right]$ represents the infinitesimal deformation of $\mathcal{M}_{2 \mathrm{~d}}$ induced by perturbing the bulk theory with $Q^{4} \Phi$. Indeed, this deformation can be computed by using the Ehresmann connection in $\mathcal{M}_{2 \mathrm{~d}-4 \mathrm{~d}}$ to identify the fibers with a fixed space $\mathcal{M}_{2 \mathrm{~d}}$ to first order in $\epsilon$, and then looking at the $\epsilon$ dependence of vectors in $T_{\epsilon}^{0,1} \mathcal{M}_{2 \mathrm{~d}}$; this is what we have computed in (3.8).

The fact that $\mu_{\Phi}$ is covariantly antiholomorphic constrains the representative in the class $\left[\mu_{\Phi}\right]$ : indeed it implies that $\mu_{\Phi}$ can be written in the form

$$
\mu_{\Phi}=g^{-1} \overline{P_{\Phi}}
$$

where $g$ is the Zamolodchikov metric on $\mathcal{M}_{2 \mathrm{~d}}$ and $P_{\Phi}$ is a holomorphic quadratic differential on $\mathcal{M}_{2 \mathrm{~d}}$ (or perhaps meromorphic if $\mathcal{M}_{2 \mathrm{~d}}$ has singularities, e.g. the punctures in class $S$ theories).

\footnotetext{
${ }^{7}$ In terms of the chiral ring coefficients $C_{I J}^{K}$ in an $\mathcal{N}=(2,2)$ theory, the integrability condition is usually written $\nabla_{I} C_{J K}^{L}=\nabla_{J} C_{I K}^{L}$. Using the metric to raise the $K$ and lower the $L$ indices it becomes $\nabla_{I} C_{J \bar{L}}^{\bar{K}}=\nabla_{J} C_{I \bar{L}}^{\bar{K}} ;$ complex conjugating gives $\nabla_{\bar{I}} \bar{C} \bar{J}_{L}^{K}=\nabla_{\bar{J}} \bar{C}_{\bar{I} L}^{K}$, which is the form we are using, with $\Phi$ standing in for the $L$ index.
} 


\section{Example: U(1) gauge theory with solenoid defect}

In this section we check our results in a very simple example: the free $\mathcal{N}=2 \mathrm{U}(1)$ gauge theory with a solenoid defect. In section 4.1 we give definitions and fix normalizations. This is followed by a computation of the deformation of the defect moduli space in section 4.2.

\subsection{Setup}

We consider the pure $\mathcal{N}=2$ theory with gauge group $\mathrm{U}(1)$, described by a free vector multiplet $\left(\phi, A_{\mu}, \lambda_{i}{ }^{\alpha}, D^{i j}\right)$. Here, $\phi$ is a complex scalar, $A_{\mu}$ is a $\mathrm{U}(1)$ gauge field, $\lambda_{i}{ }^{\alpha}$ are fermions in the doublets of $\mathfrak{s u}(2)_{L}$ and $\mathfrak{s u}(2)_{r}$, and $D^{i j}$ is an $\mathfrak{s u}(2)_{r}$ triplet of auxiliary fields,

$$
D^{i j}=\left(\begin{array}{cc}
\sqrt{2} F & \mathrm{i} D \\
\mathrm{i} D & \sqrt{2 F}
\end{array}\right) .
$$

The action is:

$$
\begin{aligned}
S_{4 \mathrm{~d}}= & \frac{1}{g^{2}} \int \mathrm{d}^{4} x\left(\frac{1}{4} F_{\mu \nu} F^{\mu \nu}-\phi \square \bar{\phi}-|F|^{2}-\frac{1}{2} D^{2}+\mathrm{i} \lambda_{i}{ }^{\alpha} \sigma_{\alpha \dot{\beta}}^{\rho} \partial_{\rho} \bar{\lambda}^{i \dot{\beta}}\right) \\
& +\frac{\mathrm{i} \vartheta}{32 \pi^{2}} \int \mathrm{d}^{4} x \widetilde{F}^{\mu \nu} F_{\mu \nu} .
\end{aligned}
$$

The supersymmetry transformations of the fields are:

$$
\begin{aligned}
& Q^{i}{ }_{\alpha} \phi=\sqrt{2} \lambda^{i}{ }_{\alpha} \\
& ; \quad \bar{Q}_{j \dot{\beta}} \bar{\phi}=\sqrt{2} \bar{\lambda}_{j \dot{\beta}} \\
& Q^{i}{ }_{\alpha} A_{\mu}=+i \sigma_{\mu \alpha \dot{\gamma}} \bar{\lambda}^{i \dot{\gamma}} \\
& ; \quad \bar{Q}_{j \dot{\beta}} A_{\mu}=i \lambda_{j}^{\gamma} \sigma_{\mu \gamma \dot{\beta}} \\
& Q^{i}{ }_{\alpha} \bar{\lambda}^{k \dot{\gamma}}=-i \sqrt{2} \epsilon^{\dot{\alpha} \dot{\gamma}} \epsilon^{i k} \sigma_{\alpha \dot{\alpha}}^{\mu} \partial_{\mu} \bar{\phi} \\
& ; \quad \bar{Q}_{j \dot{\beta}} \lambda_{k}{ }^{\gamma}=i \sqrt{2} \epsilon_{j k} \epsilon^{\gamma \alpha} \sigma_{\alpha \dot{\beta}}^{\mu} \partial_{\mu} \phi \\
& Q^{i}{ }_{\alpha} \lambda_{j}{ }^{\gamma}=\delta_{\alpha}{ }^{\gamma} D_{j}{ }^{i}-\delta^{i}{ }_{j}\left(\epsilon \sigma^{\rho \sigma} \epsilon\right)^{\gamma}{ }_{\alpha} F_{\rho \sigma} \quad ; \quad \bar{Q}_{j \dot{\beta}} \bar{\lambda}^{i \dot{\gamma}}=-\delta^{\dot{\gamma}}{ }_{\dot{\beta}} D_{j}{ }^{i}+\left(\epsilon \bar{\sigma}^{\rho \sigma} \epsilon\right)_{\dot{\beta}}{ }^{\dot{\gamma}} \delta_{j}{ }^{i} F_{\rho \sigma}
\end{aligned}
$$

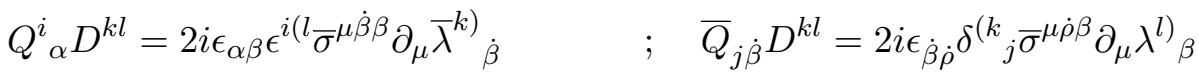

We consider the chiral operator $\Phi=\phi^{2}$, and its marginal descendant given by:

$$
Q^{4} \phi^{2}(x)=\frac{1}{8 \pi \mathrm{i}}\left(-\phi \square \bar{\phi}-\mathrm{i} \lambda_{i}^{\alpha} \sigma_{\alpha \dot{\beta}}^{\rho} \partial_{\rho} \bar{\lambda}^{i \dot{\beta}}+\frac{1}{4} F_{\mu \nu} F^{\mu \nu}-\frac{1}{4} \widetilde{F}^{\mu \nu} F_{\mu \nu}-\frac{1}{4} D_{i j} D^{i j}\right) .
$$

Here $\widetilde{F}^{\mu \nu}=\frac{1}{2} \epsilon^{\mu \nu \rho \sigma} F_{\rho \sigma}$, and in what follows, we write $F^{ \pm}=F \pm \widetilde{F}$. The parameter associated to the marginal operator is the complexified gauge coupling $\tau=\frac{4 \pi \mathrm{i}}{g^{2}}+\frac{\vartheta}{2 \pi}$.

We insert a $\frac{1}{2}$-BPS defect on $\mathbb{R}_{x^{1}=x^{2}=0}$ defined by the action [16]

$$
S_{2 \mathrm{~d}}=-\int_{\mathbb{R}^{2}} \mathrm{~d}^{2} x\left(\frac{4 \pi \alpha}{g^{2}}\left(F_{12}+D\right)+\left(\eta+\frac{\vartheta}{2 \pi} \alpha\right) \mathrm{i} F_{34}\right) .
$$

This defect behaves like a solenoid: its only effect is to create an Aharonov-Bohm phase. The first term gives the boundary condition $A=\alpha \mathrm{d} \theta$ to the gauge field around $\mathbb{R}_{x^{1}=x^{2}=0}^{2}$. The gauge invariant information is captured by the holonomy $\mathrm{e}^{2 \pi \mathrm{i} \alpha}$, and hence $\alpha \sim \alpha+1$. The second term is the $2 \mathrm{~d}$ theta term, and hence $\eta \sim \eta+1$.

The bulk chiral operator $\phi$ and anti-chiral operator $\bar{\phi}$ are also chiral and anti-chiral, respectively, with respect to the defect supersymmetry algebra. The second descendants 
$Q^{2} \phi=\frac{1}{2 \sqrt{2}} Q^{1}{ }_{1} Q^{2}{ }_{2} \phi$ and $\bar{Q}^{2} \bar{\phi}=\frac{1}{2 \sqrt{2}} \bar{Q}_{2 \dot{2}} \bar{Q}_{1 \mathrm{i}} \bar{\phi}$ are marginal operators on the defect, explicitly given by

$$
\begin{aligned}
Q^{2} \phi & =\frac{\mathrm{i}}{2}\left(D+F_{12}-F_{34}\right), \\
\bar{Q}^{2} \bar{\phi} & =-\frac{\mathrm{i}}{2}\left(D+F_{12}+F_{34}\right) .
\end{aligned}
$$

The coupling constant associated to $Q^{2} \phi$ is the FI parameter

$$
z=\eta+\tau \alpha
$$

This parameter lies in the complex torus $\mathcal{M}_{2 \mathrm{~d}}=\mathbb{C} / \Lambda_{\tau}$, where $\Lambda_{\tau}=\mathbb{Z} \oplus \tau \mathbb{Z}$. A deformation of the marginal coupling $\tau$ thus induces a deformation of the complex structure on $\mathcal{M}_{2 \mathrm{~d}}$.

\subsection{Complex deformation}

We consider the bulk chiral operator $\Phi=\phi^{2}$ and defect chiral operator $\Sigma=\phi$. Their OPE is

$$
\Phi(x) \bar{\Sigma}(0)=-\frac{g^{2}}{2 \pi^{2}|x|^{2}} \Sigma(0)+\cdots
$$

Comparing this with (1.7) we have

$$
\mu_{\Phi}(\bar{\Sigma})=\frac{g^{2}}{8 \pi \mathrm{i}} \Sigma=\frac{1}{\tau-\bar{\tau}} \Sigma .
$$

Identifying $\bar{\Sigma} \rightarrow \partial_{\bar{z}}$ and $\Sigma \rightarrow \partial_{z}$, this gives $\mu_{\Phi} \in \Omega^{0,1}\left(\mathcal{M}_{2 \mathrm{~d}}, T \mathcal{M}_{2 \mathrm{~d}}\right)$ as

$$
\left(\mu_{\Phi}\right)_{\bar{z}}^{z}=\frac{1}{\tau-\bar{\tau}} .
$$

This tensor indeed represents the deformation of $\mathcal{M}_{2 \mathrm{~d}}$ corresponding to $Q^{4} \Phi$, i.e. to $\partial_{\tau}$. Indeed, changing $\tau$ to $\tau+\delta \tau$ can be realized explicitly by changing the complex coordinate on $\mathcal{M}_{2 \mathrm{~d}}$ from $z$ to $z^{\prime}=z+\alpha \delta \tau$, and under this coordinate change we have

$$
\frac{\partial}{\partial \overline{z^{\prime}}}=\frac{\partial}{\partial \bar{z}}+\frac{\delta \tau}{\tau-\bar{\tau}} \frac{\partial}{\partial z}
$$

Comparing this with (C.6) we see that the deformation can be represented by the tensor $\mu_{\bar{z}}^{z}=\frac{1}{\tau-\bar{\tau}}$, which indeed matches the $\mu_{\Phi}$ we computed above. This verifies that our prescription for the deformation of $\mathcal{M}_{2 \mathrm{~d}}$ induced by a bulk deformation works in this case.

\section{A Four-dimensional superconformal algebra}

The four-dimensional conformal algebra is generated by translation $\left(P_{\mu}\right)$, special conformal transformation $\left(K_{\mu}\right)$, rotations $\left(J_{\mu \nu}\right)$ and dilatation $(D)$. When extended to have $\mathcal{N}=2$ supersymmetry, the algebra has eight Poincaré supercharges $\left(Q_{\alpha}^{i}, \bar{Q}_{j \dot{\beta}}\right)$ and eight special conformal supercharges $\left(S_{i}{ }^{\alpha}, \bar{S}^{j \dot{\beta}}\right)$. The latin indices denote the components of $\mathfrak{s u}(2)_{r}$ doublet, and undotted (dotted) Greek indices denote the components of $\mathfrak{s u}(2)_{L}\left(\mathfrak{s u}(2)_{R}\right)$ 
doublets. We raise and lower indices of each $\mathfrak{s u}(2)$ doublet through the two-dimensional Levi-Civita tensor $\epsilon_{i j}$, where we use the convention $\epsilon_{21}=\epsilon^{12}=1$. The generators of $\mathfrak{s u}(2)_{L} \oplus \mathfrak{s u}(2)_{R}$ are related to $J_{\mu \nu}$ through the following relations. ${ }^{8}$

$$
J_{\alpha}^{\beta}=-\frac{i}{4}\left(\sigma^{\mu} \bar{\sigma}^{\nu}\right)_{\alpha}{ }^{\beta} J_{\mu \nu} ; \quad \bar{J}_{\dot{\beta}}^{\dot{\alpha}}=\frac{i}{4}\left(\bar{\sigma}^{\mu} \sigma^{\nu}\right)_{\dot{\beta}}^{\dot{\alpha}} J_{\mu \nu}
$$

The nonzero anti-commutators of odd generators are as follows.

$$
\begin{aligned}
\left\{Q^{i}{ }_{\alpha}, \bar{Q}_{j \dot{\beta}}\right\} & =2 \delta^{i}{ }_{j} \sigma_{\alpha \dot{\beta}}^{\mu} P_{\mu} \\
\left\{S_{i}{ }^{\alpha}, \bar{S}^{j \dot{\beta}}\right\} & =2 \delta_{i}{ }^{j} \bar{\sigma}^{\mu \dot{\beta} \alpha} K_{\mu} \\
\left\{Q^{i}{ }_{\alpha}, S_{j}{ }^{\beta}\right\} & =4\left(\delta^{i}{ }_{j} J_{\alpha}{ }^{\beta}-\delta_{\alpha}{ }^{\beta} R^{i}{ }_{j}+\frac{1}{2} \delta^{i}{ }_{j} \delta_{\alpha}{ }^{\beta} D\right) \\
\left\{\bar{Q}_{j \dot{\beta}}, \bar{S}^{i \dot{\alpha}}\right\} & =4\left(\delta^{i}{ }_{j} \bar{J}^{\dot{\alpha}}{ }_{\dot{\beta}}+\delta^{\dot{\alpha}}{ }_{\dot{\beta}} R^{i}{ }_{j}+\frac{1}{2} \delta^{i}{ }_{j} \delta^{\dot{\alpha}}{ }_{\dot{\beta}} D\right)
\end{aligned}
$$

Here and throughout the paper, $\sigma^{\mu}=(\vec{\sigma}, i), \bar{\sigma}^{\mu}=(-\vec{\sigma}, i)$. The components of $\vec{\sigma}$ are Pauli matrices.

$$
\begin{aligned}
{\left[J_{\alpha}{ }^{\beta}, Q^{i}{ }_{\gamma}\right] } & =\delta_{\gamma}{ }^{\beta} Q^{i}{ }_{\alpha}-\frac{1}{2} \delta_{\alpha}{ }^{\beta} Q^{i}{ }_{\gamma} \\
{\left[\bar{J}^{\dot{\alpha}}{ }_{\dot{\beta}}, \bar{Q}_{j \dot{\gamma}}\right] } & =\delta^{\dot{\alpha}}{ }_{\dot{\gamma}} \bar{Q}_{j \dot{\beta}}-\frac{1}{2} \delta^{\dot{\alpha}}{ }_{\dot{\beta}} \bar{Q}_{j \dot{\gamma}} \\
{\left[J_{\alpha}{ }^{\beta}, S_{i}{ }^{\gamma}\right] } & =-\delta_{\alpha}{ }^{\gamma} S_{i}{ }^{\beta}+\frac{1}{2} \delta_{\alpha}{ }^{\beta} S_{i}{ }^{\gamma} \\
{\left[\bar{J}^{\dot{\alpha}}{ }_{\dot{\beta}}, \bar{S}^{i \dot{\gamma}}\right] } & =-\delta^{\dot{\gamma}} \bar{\beta}^{i \dot{\alpha}}+\frac{1}{2} \delta^{\dot{\alpha}}{ }_{\dot{\beta}} \bar{S}^{i \dot{\gamma}}
\end{aligned}
$$

The nonzero commutators of supercharges with translations and special conformal transformations are as follows.

$$
\begin{aligned}
{\left[K_{\mu}, Q^{i}{ }_{\alpha}\right] } & =-\left(\sigma_{\mu}\right)_{\alpha \dot{\alpha}} \bar{S}^{i \dot{\alpha}}, & {\left[K_{\mu}, \bar{Q}_{j \dot{\beta}}\right] } & =-\left(\sigma_{\mu}\right)_{\alpha \dot{\beta}} \\
{\left[P_{\mu}, \bar{S}^{i \dot{\alpha}}\right] } & =\left(\bar{\sigma}_{\mu}\right)^{\dot{\alpha} \beta} Q^{i}{ }_{\beta}, & {\left[P_{\mu}, S_{i}{ }^{\alpha}\right] } & =\left(\bar{\sigma}_{\mu}\right)^{\dot{\alpha} \alpha} \bar{Q}_{i \dot{\alpha}}
\end{aligned}
$$

The $\mathfrak{s u}(2)_{r}$ algebra has ladder operators $R_{ \pm}$and Cartan generator $R_{3}$. The supercharges $Q^{1}{ }_{\alpha}, \bar{Q}_{2 \dot{\alpha}}, S_{2}{ }^{\alpha}$ and $\bar{S}^{1 \dot{\alpha}}$ have $R_{3}$-charge $+\frac{1}{2}$; while $Q^{2}{ }_{\alpha}, \bar{Q}_{1 \dot{\alpha}}, S_{1}{ }^{\alpha}$ and $\bar{S}^{2 \dot{\alpha}}$ have $R_{3}$-charge $-\frac{1}{2}$. Lastly, the scaling dimensions and $\mathfrak{u}(1)_{r}$ charges of all supercharges are as follows.

$$
\begin{gathered}
{\left[D, Q^{i}{ }_{\alpha}\right]=\left[r, Q^{i}{ }_{\alpha}\right]=\frac{1}{2} Q^{i}{ }_{\alpha}} \\
-\left[D, \bar{Q}_{j \dot{\beta}}\right]=\left[r, \bar{Q}_{j \dot{\beta}}\right]=-\frac{1}{2} \bar{Q}_{j \dot{\beta}} \\
{\left[D, S_{i}{ }^{\alpha}\right]=\left[r, S_{i}{ }^{\alpha}\right]=-\frac{1}{2} S_{i}{ }^{\alpha}} \\
-\left[D, \bar{S}^{i \dot{\alpha}}\right]=\left[r, \bar{S}^{i \dot{\alpha}}\right]=\frac{1}{2} \bar{S}^{i \dot{\alpha}}
\end{gathered}
$$

\footnotetext{
${ }^{8}$ Our conventions compare with the conventions of [17] as follows: for $x \in\left\{\bar{S}^{i \dot{\alpha}}, K_{\mu}, \bar{J}^{\dot{\alpha}}{ }_{\dot{\beta}}\right\}, x^{\text {here }}=$ $-x^{\text {there }}$. Also, $D^{\text {here }}=-i D^{\text {there }}$.
} 


\section{B Two-dimensional superconformal algebra}

Now we discuss the superconformal algebra in two dimensions with $\mathcal{N}=(2,2)$ supersymmetry. We take the surface defect to be present at $x^{1}=x^{2}=0$. The conformal algebra in two dimensions in terms of holomorphic operators $\left(L_{n}\right)$ and anti-holomorphic operators $\left(\bar{L}_{n}\right)$ for $n=-1,0,+1$.

$$
\begin{aligned}
i P_{4} & =L_{-1}+\bar{L}_{-1}, & P_{3} & =L_{-1}-\bar{L}_{-1} \\
i K_{4} & =L_{1}+\bar{L}_{1}, & & K_{3}=\bar{L}_{1}-L_{1} \\
D & =L_{0}+\bar{L}_{0}, & J_{34} & =L_{0}-\bar{L}_{0}
\end{aligned}
$$

In an $\mathcal{N}=(2,2)$ superconformal algebra, there are eight fermionic generators which we notate as $G_{1 / 2}^{ \pm}, G_{-1 / 2}^{ \pm}, \bar{G}_{1 / 2}^{ \pm}$and $\bar{G}_{-1 / 2}^{ \pm}$. The nonzero (anti-) commutation relations of the operators are as follows.

$$
\begin{aligned}
{\left[L_{n}, G_{r}^{ \pm}\right] } & =\left(\frac{n}{2}-r\right) G_{n+r}^{ \pm} \\
{\left[\bar{L}_{n}, \bar{G}_{r}^{ \pm}\right] } & =\left(\frac{n}{2}-r\right) \bar{G}_{n+r}^{ \pm} \\
\left\{G_{r}^{+}, G_{s}^{-}\right\} & =L_{r+s}+\frac{r-s}{2} J_{r+s} \\
\left\{\bar{G}_{r}^{+}, \bar{G}_{s}^{-}\right\} & =\bar{L}_{r+s}+\frac{r-s}{2} \bar{J}_{r+s}
\end{aligned}
$$

The algebra has $\mathfrak{u}(1)_{L} \oplus \mathfrak{u}(1)_{R}$ R-symmetry with generators $J_{0}, \bar{J}_{0}$.

$$
\left[J_{0}, G_{ \pm 1 / 2}^{a}\right]=a G_{ \pm 1 / 2}^{a}, \quad\left[\bar{J}_{0}, \bar{G}_{ \pm 1 / 2}^{a}\right]=a \bar{G}_{ \pm 1 / 2}^{a}
$$

Comparison with four-dimensional $\mathcal{N}=2$ superconformal algebra gives the following identification of operators.

$$
\begin{aligned}
& G_{-1 / 2}^{+}=\frac{Q^{1}{ }_{1}}{2}, \quad G_{-1 / 2}^{-}=\frac{\bar{Q}_{\dot{1} 1}}{2}, \quad G_{+1 / 2}^{-}=\frac{S_{1}{ }^{1}}{2}, \quad G_{+1 / 2}^{+}=\frac{\bar{S}^{11}}{2} \\
& \bar{G}_{-1 / 2}^{-}=\frac{Q^{2} 2}{2}, \quad \bar{G}_{-1 / 2}^{+}=\frac{\bar{Q}_{\dot{2} 2}}{2}, \quad \bar{G}_{+1 / 2}^{+}=\frac{S_{2}{ }^{2}}{2}, \quad \bar{G}_{+1 / 2}^{-}=\frac{\bar{S}^{2 \dot{2}}}{2} \\
& J_{0}=2 R_{3}+J_{12}+r, \quad \bar{J}_{0}=2 R_{3}+J_{12}-r
\end{aligned}
$$

We choose the following conventions for the vector and axial R-charges: $J_{V}=J_{0}-\bar{J}_{0}$ and $J_{A}=J_{0}+\bar{J}_{0}$. With this convention, the bulk chiral primary is also a chiral primary with respect to the defect superconformal algebra.

\section{Deformations of complex manifolds}

Here we review some basic facts about deformations of complex manifolds; see e.g. [18] for a more complete treatment. 
Let $M$ be a manifold of real dimension $2 n$. A complex structure on $M$ is a section $I \in \operatorname{End}(T M)$ such that $I^{2}=-1$ and the Nijenhuis tensor $N_{I}$ vanishes. When the latter condition is not necessarily satisfied, $I$ is an almost complex structure on $M$.

The data of an almost complex structure $I$ is uniquely determined by the decomposition

$$
T_{\mathbb{C}} M=T^{1,0} M \oplus T^{0,1} M
$$

such that $\left.I\right|_{T^{1,0} M}=\mathrm{i}$ and $\left.I\right|_{T^{0,1} M}=-\mathrm{i}$. Now suppose we have a family of almost complex structures $I_{\epsilon}$ on $M$, parameterized by a variable $\epsilon$. Then we have a family of decompositions with respect to $I_{\epsilon}$,

$$
T_{\mathbb{C}} M=T_{\epsilon}^{1,0} M \oplus T_{\epsilon}^{0,1} M
$$

For sufficiently small $\epsilon$, the deformation $I_{\epsilon}$ may be encoded by a map

$$
J_{\epsilon}: T_{0}^{0,1} M \rightarrow T_{0}^{1,0} M
$$

such that if $v \in T_{0}^{0,1} M$ then $v+\epsilon J_{\epsilon} v \in T_{\epsilon}^{0,1} M$. In this paper, we will only be interested in studying deformations of complex structure to first order in $\epsilon$. To this order, the requirement that the Nijenhuis tensor of $I_{\epsilon}$ vanishes is equivalent to the requirement that $J_{\epsilon} \in \Omega_{0}^{0,1}(M, T M)$ is $\bar{\partial}$-closed.

We say that two complex structures are isomorphic if there exists a self-diffeomorphism of $M$ that maps one to the other. Given a one-parameter family of diffeomorphisms $F_{\epsilon}$, we obtain a global vector field on $M, \frac{\mathrm{d} F_{\epsilon}}{\mathrm{d} \epsilon} \in \Omega^{0}(T M)$. Conversely, given a vector field on $M$, we get an infinitesimal diffeomorphism of $M$. Pushing the complex structure $I$ forward through $F_{\epsilon}$ gives a new complex structure $I_{\epsilon}=\mathrm{d} F_{\epsilon} \circ I\left(\mathrm{~d} F_{\epsilon}\right)^{-1}$, isomorphic to the original one, with the first-order deformation

$$
J_{\epsilon}=\bar{\partial}\left(\left(\frac{\mathrm{d} F_{\epsilon}}{\mathrm{d} \epsilon}\right)^{1,0}\right) .
$$

Such a $J_{\epsilon}$ is thus regarded as a trivial first-order deformation. Thus altogether the space $\operatorname{Def}(X)$ of first-order deformations of a complex manifold $X$ is isomorphic to the Dolbeault cohomology $H^{0,1}(X, T X)$.

In local coordinates on $X$, a representative $\mu$ may be written as

$$
\mu=\sum_{a, b=1}^{n} \mu_{\bar{a}}^{b} \mathrm{~d} \bar{z}^{a} \frac{\partial}{\partial z^{b}}
$$

Through the map (C.3) the antiholomorphic tangent vectors in the deformed complex structure $I_{\epsilon}$ are given as

$$
\left(\frac{\partial}{\partial \bar{z}^{a}}\right)_{\epsilon}=\frac{\partial}{\partial \bar{z}^{a}}+\epsilon \mu_{\bar{a}}^{b} \frac{\partial}{\partial z^{b}}
$$

\section{The covariant derivative of the supercurrent}

\section{D.1 Computation}

In this section we give the computation leading to (3.6). To simplify notation here we suppress some indices, writing $\bar{J}^{\mu}$ for the supercurrent $\bar{J}_{I \dot{\alpha}}^{\mu}$. 
The idea of the computation is a bit indirect. We first consider the extension of $\bar{J}^{\mu}$ to a section $\bar{J}_{\text {cc }}^{\mu}$ of the bundle of operators over the family of deformed theories, to first order in $\epsilon$, obeying $\nabla_{\epsilon} \bar{J}_{\mathrm{cc}}^{\mu}=0$. The key point is that $\bar{J}_{\mathrm{cc}}^{\mu}$ is not an allowed supercurrent, because it is not conserved at $\epsilon \neq 0$. We calculate the amount by which conservation of $\bar{J}_{\text {cc }}^{\mu}$ is violated, by writing out the inner product

$$
\partial_{\mu}\left\langle\Psi(\infty) \bar{J}_{\text {cc }}^{\mu}(x)\right\rangle
$$

between $\partial_{\mu} \bar{J}_{\text {cc }}^{\mu}$ and a general operator $\Psi$ that is also extended in a covariantly constant manner. To first order in $\epsilon$ we have

$$
\partial_{\mu}\left\langle\Psi(\infty) \bar{J}_{\text {cc }}^{\mu}(x)\right\rangle=-\epsilon \partial_{\mu} \int \mathrm{d}^{4} y\left\langle\Psi(\infty) \Phi^{(4)}(y) \bar{J}^{\mu}(x)\right\rangle
$$

Formally this would seem to be zero because we can change variables to $y^{\prime}=y-x$ in the integral, so that the integral is $x$-independent. But we will argue now that it is not actually zero once we take care of regularizations.

For this we need the OPE between $\Phi^{(4)}$ and $\bar{J}^{\mu}$ :

$$
\bar{J}^{\mu}(x) \Phi^{(4)}(0)=\frac{1}{2 \pi^{2}} \frac{x^{\mu}}{|x|^{4}} \bar{Q} \Phi^{(4)}(0)+\cdots
$$

where $\cdots$ denotes operators of dimension different from $\frac{9}{2}$, and again we suppress indices, writing $\bar{Q}$ for $\bar{Q}_{I \dot{\alpha}}$. Inserting this OPE in our integral gives

$$
\partial_{\mu}\left\langle\Psi(\infty) \bar{J}_{\mathrm{cc}}^{\mu}(x)\right\rangle=\frac{1}{2 \pi^{2}} \epsilon \partial_{\mu} \int \mathrm{d}^{4} y \frac{(y-x)^{\mu}}{|y-x|^{4}}\left\langle\Psi(\infty) \bar{Q} \Phi^{(4)}(x)\right\rangle+\cdots
$$

The domain $R_{x}$ of integration here is a big ball $|y|<M$, excluding a small ball around $y=x$. Changing variable to $y^{\prime}=y-x$, the domain $R_{x}^{\prime}$ becomes a ball $\left|y^{\prime}+x\right|<M$ with a small ball around $y^{\prime}=0$ deleted, and

$$
\partial_{\mu}\left\langle\Psi(\infty) \bar{J}_{\mathrm{cc}}^{\mu}(x)\right\rangle=\frac{1}{2 \pi^{2}} \epsilon \partial_{\mu}\left[\left\langle\Psi(\infty) \bar{Q} \Phi^{(4)}(x)\right\rangle \int_{R_{x}^{\prime}} \mathrm{d}^{4} y^{\prime} \frac{y^{\prime \mu}}{\left|y^{\prime}\right|^{4}}\right]+\cdots
$$

If we specialize to the case where $\Psi$ is an operator of dimension $\frac{9}{2}$, then the extra contributions $\cdots$ vanish, and also we can replace $x$ by 0 in the vev, getting

$$
\partial_{\mu}\left\langle\Psi(\infty) \bar{J}_{\mathrm{cc}}^{\mu}(x)\right\rangle=\frac{1}{2 \pi^{2}} \epsilon\left\langle\Psi \mid \bar{Q} \Phi^{(4)}\right\rangle \partial_{\mu}\left[\int_{R_{x}^{\prime}} \mathrm{d}^{4} y^{\prime} \frac{y^{\prime \mu}}{\left|y^{\prime}\right|^{4}}\right]
$$

Differentiating the integral gives $-2 \pi^{2}$ (see next section). This equation holds for any operator $\Psi$ of dimension $\frac{9}{2}$ so we get $^{9}$

$$
\partial_{\mu} \bar{J}_{\mathrm{cc}}^{\mu}=-\epsilon \bar{Q} \Phi^{(4)} .
$$

\footnotetext{
${ }^{9}$ We expect that $\nabla_{\epsilon}$ only mixes operators with the same scaling dimension. This result has been derived for two dimensional conformal field theories in [7] where this connection is called the $\bar{c}$ connection. Furthermore, using the operator-state correspondence, $\nabla_{\epsilon}$ is related to the Berry connection over the vector bundle of Hilbert spaces in [13] where the latter only mixes states with equal energy. These results indicate that equation (D.7) does not get any correction from operators of other scaling dimensions.
} 
How do we interpret (D.7)? It means that $\bar{J}_{\text {cc }}^{\mu}$ is not conserved, but it also shows us how to fix the problem: if we find an operator $\mathcal{O}^{\mu}$ obeying

$$
\partial_{\mu} \mathcal{O}^{\mu}=\bar{Q} \Phi^{(4)}
$$

then we could consider a new section

$$
\bar{J}_{\text {cons }}^{\mu}=\bar{J}_{\text {cc }}^{\mu}+\epsilon \mathcal{O}^{\mu}
$$

and then $\bar{J}_{\text {cons }}^{\mu}$ is a conserved supercurrent, obeying ${ }^{10}$

$$
\nabla_{\epsilon} \bar{J}_{\text {cons }}^{\mu}=\mathcal{O}^{\mu}
$$

Moreover, the condition (D.8) has a canonical solution: we can take $\mathcal{O}=\Phi^{(3)}$. Any other solution must differ from $\Phi^{(3)}$ by a conserved supercurrent, which can only be of the form $c \bar{J}^{\mu}$ for some scalar $c$ (since we assume the theory has exactly $\mathcal{N}=2$ supersymmetry, not more). Thus finally

$$
\nabla_{\epsilon} \bar{J}_{\mathrm{cons}}^{\mu}=\left(\Phi^{(3)}\right)^{\mu}+c \bar{J}^{\mu}
$$

which is the desired (3.6).

\section{D.2 An integral}

In this section we relabel $y^{\prime} \rightarrow y$ and $R^{\prime} \rightarrow R$, and we work in $d$ dimensions. Fix parameters $M, \epsilon$ and define the region

$$
R_{x}=\{y:|y+x|<M,|y|>\epsilon\}
$$

Then let

$$
I^{\mu}(x)=\int_{R_{x}} \mathrm{~d}^{d} y \frac{y^{\mu}}{|y|^{d}} .
$$

For a fixed $\mu$, the derivative $\partial_{\mu} I^{\mu}$ can be computed as follows. We shift $x$ by (an infinitesimal) $t$ units in the positive $\mu$ direction. Consider the side $y^{\mu}>0$ of $R_{x}$. On this side, the size of $R_{x}$ is decreased by translating the frontier $y$ by $-t$ in the $\mu$ direction. On the other side we are similarly increasing the size of $R_{x}$. On each side we can approximate the integrand by its value on the sphere.

The change in $I^{\mu}$ can thus be represented as an integral over the coordinates other than $\mu$, which span a ball $B^{d-1}$, with radial coordinate $r$ and the usual area element $\mathrm{d} A_{d-1}$ :

$$
\begin{aligned}
\delta I^{\mu} & =2 \int_{B_{M}^{d-1}} \frac{\sqrt{M-r^{2}}}{M^{d}}(-t) \mathrm{d} A_{d-1} \\
& =\left(-t M^{-d}\right) \int_{0}^{M} \mathrm{~d} r \int_{S_{r}^{d-2}} 2 \sqrt{M-r^{2}} \mathrm{~d} A_{d-1} \\
& =\left(-t M^{-d}\right) \operatorname{Vol}\left(B_{M}^{d}\right) \\
& =-t \operatorname{Vol}\left(B^{d}\right) .
\end{aligned}
$$

\footnotetext{
${ }^{10}$ The derivative $\nabla_{\epsilon}$ is computed at $\epsilon=0$. Therefore, we do not write down $\epsilon \nabla_{\epsilon} \mathcal{O}^{\mu}$ in equation (D.10).
} 
Thus we have with $\mu$ fixed

$$
\partial_{\mu} I^{\mu}=-\operatorname{Vol}\left(B^{d}\right)
$$

and summing over the index $\mu$,

$$
\partial_{\mu} I^{\mu}=-d \operatorname{Vol}\left(B^{d}\right)=-\operatorname{Vol}\left(S^{d-1}\right) .
$$

\section{D.3 With a surface defect}

In the presence of a $\frac{1}{2}$-BPS surface defect $\mathrm{S}(P)$, in addition to the bulk supercurrent $\bar{J}^{\mu}$ there is also the defect supercurrent $\bar{J}_{\mathrm{d}}^{\mu}$, defined on $P$, with the property that (loosely speaking) the combined $\bar{J}+\delta(P) \bar{J}_{\mathrm{d}}$ is conserved. One sharp way to express this conservation is to say that $\partial_{\mu} \bar{J}_{\mathrm{d}}^{\mu}$ has to cancel the delta-function divergence of the bulk supercurrent $\bar{J}^{\mu}$ in the normal directions, which we can measure as the flux through a small circle linking the defect: the pair $\left(\bar{J}, \bar{J}_{\mathrm{d}}\right)$ has to obey

$$
\partial_{\mu} \bar{J}_{\mathrm{d}}^{\mu}(y)=\lim _{r \rightarrow 0} r \oint_{S_{r}^{1}(y)} \bar{J}^{r}(x) \mathrm{d} \theta .
$$

Now, in parallel to the discussion of $\nabla_{\epsilon} \bar{J}^{\mu}$ in section D.1, we may consider the covariant derivative $\nabla_{\epsilon} \bar{J}_{\mathrm{d}}^{\mu}$. A computation parallel to that of section D.1 gives no order $\epsilon$ contribution to $\partial_{\mu} \bar{J}_{\mathrm{d}, \mathrm{cc}}^{\mu}$; thus to first order in $\epsilon$ we have the same equation as at $\epsilon=0$,

$$
\partial_{\mu} \bar{J}_{\mathrm{d}, \mathrm{cc}}^{\mu}(y)=\lim _{r \rightarrow 0} r \oint_{S_{r}^{1}(y)} \bar{J}_{\mathrm{cc}}^{r}(x) \mathrm{d} \theta .
$$

Replacing $\bar{J}_{\text {cc }}^{r}$ in favor of the bulk conserved supercurrent $\bar{J}_{\text {cons }}^{r}$ this becomes

$$
\partial_{\mu} \bar{J}_{\mathrm{d}, \mathrm{cc}}^{\mu}(y)=\lim _{r \rightarrow 0} r \oint_{S_{r}^{1}(y)}\left(\bar{J}_{\text {cons }}^{r}(x)-\epsilon\left(\Phi^{(3)}\right)^{r}\right) \mathrm{d} \theta .
$$

Thus, if we define $\bar{J}_{\mathrm{d} \text {,cons }}^{\mu}$ by

$$
\bar{J}_{\mathrm{d}, \mathrm{cons}}^{\mu}=\bar{J}_{\mathrm{d}, \mathrm{cc}}^{\mu}+\epsilon C^{\mu},
$$

where $C^{\mu}$ is a defect operator obeying

$$
\partial_{\mu} C^{\mu}(y)=r \lim _{r \rightarrow 0} \oint_{S_{r}^{1}(y)}\left(\Phi^{(3)}\right)^{r} \mathrm{~d} \theta,
$$

then the pair $\left(\bar{J}_{\text {cons }}^{\mu}, \bar{J}_{\text {d,cons }}^{\mu}\right)$ obeys the desired (D.20).

To determine $C^{\mu}$ we now examine the right side of (D.24). It is nonzero just if there is a divergence of order $1 / r$ in the OPE between $\left(\Phi^{(3)}\right)^{r}$ and the surface defect $\mathrm{S}(P)$. For notational convenience let us consider just the single component $\left(\Phi^{(3)}\right)_{2 \dot{2}}^{r}$. Using (3.13) and the fact that $\mathrm{S}(P)$ preserves $Q^{1}{ }_{1}$ and $Q^{2}{ }_{2}$, this OPE takes the form

$$
\left(\Phi^{(3)}\right)_{22}^{r} \mathrm{~S}(P)=\mathrm{e}^{-\mathrm{i} \theta} Q^{1}{ }_{1} Q^{2}{ }_{2}\left(Q^{1}{ }_{2} \Phi(x) \mathrm{S}(P)\right) .
$$

$\Phi(x)$ has $\Delta=2, r=-2$, and thus $Q^{1}{ }_{2} \Phi(x)$ has $\Delta=\frac{5}{2}, r=-\frac{3}{2}$. An operator with this $R$-charge must have dimension at least $\frac{3}{2}$; the most singular term in the OPE is thus of the form

$$
\left(\Phi^{(3)}\right)_{2 \dot{2}}^{r} \mathrm{~S}(P)=\frac{1}{r} Q^{1}{ }_{1} Q^{2}{ }_{2}(\xi(y) \mathrm{S}(P))+\cdots
$$


where $y$ is the projection of $x$ to $P$. The defect operator $\xi$ saturates $R$-charge bounds on both sides (in the 2-d SCFT notation it has $\left(L_{0}, j_{0}\right)=\left(\frac{1}{2},-1\right)$ and $\left.\left(\bar{L}_{0}, \bar{j}_{0}\right)=(1,2)\right)$, and thus it is a superconformal primary. Combining (D.26) with (D.24) we have

$$
\partial_{\mu} C^{\mu}=2 \pi Q_{1}^{1} Q_{2}^{2} \xi
$$

However, the fact that $\xi$ is a superconformal primary implies $Q^{1}{ }_{1} Q^{2}{ }_{2} \xi$ is orthogonal to all conformal descendants; thus we conclude that $Q^{1}{ }_{1} Q^{2}{ }_{2} \xi=0$. It follows that we can take $C^{\mu}=0$, i.e. $\nabla_{\epsilon} \bar{J}_{\mathrm{d}, \mathrm{cons}}^{\mu}=0$.

Open Access. This article is distributed under the terms of the Creative Commons Attribution License (CC-BY 4.0), which permits any use, distribution and reproduction in any medium, provided the original author(s) and source are credited.

\section{References}

[1] L.F. Alday, D. Gaiotto, S. Gukov, Y. Tachikawa and H. Verlinde, Loop and surface operators in $N=2$ gauge theory and Liouville modular geometry, JHEP 01 (2010) 113 [arXiv: 0909.0945] [INSPIRE].

[2] D. Gaiotto, Surface operators in $N=24$ d gauge theories, JHEP 11 (2012) 090 [arXiv: 0911.1316] [INSPIRE].

[3] D. Gaiotto, $N=2$ dualities, JHEP 08 (2012) 034 [arXiv:0904.2715] [INSPIRE].

[4] N. Seiberg, Observations on the moduli space of superconformal field theories, Nucl. Phys. B 303 (1988) 286 [INSPIRE].

[5] D. Kutasov, Geometry on the space of conformal field theories and contact terms, Phys. Lett. B 220 (1989) 153 [INSPIRE].

[6] K. Ranganathan, Nearby CFTs in the operator formalism: the role of a connection, Nucl. Phys. B 408 (1993) 180 [hep-th/9210090] [INSPIRE].

[7] K. Ranganathan, H. Sonoda and B. Zwiebach, Connections on the state space over conformal field theories, Nucl. Phys. B 414 (1994) 405 [hep-th/9304053] [INSPIRE].

[8] V.V. Fock and A. Thomas, Higher complex structures, arXiv:1812.11199.

[9] K. Papadodimas, Topological anti-topological fusion in four-dimensional superconformal field theories, JHEP 08 (2010) 118 [arXiv:0910.4963] [INSPIRE].

[10] G.W. Moore, Finite in all directions, hep-th/9305139 [INSPIRE].

[11] M. Baggio, V. Niarchos and K. Papadodimas, $t t^{*}$ equations, localization and exact chiral rings in $4 d \mathcal{N}=2$ SCFTs, JHEP 02 (2015) 122 [arXiv: 1409.4212] [INSPIRE].

[12] E. Gerchkovitz, J. Gomis, N. Ishtiaque, A. Karasik, Z. Komargodski and S.S. Pufu, Correlation functions of Coulomb branch operators, JHEP 01 (2017) 103 [arXiv: 1602.05971] [INSPIRE].

[13] M. Baggio, V. Niarchos and K. Papadodimas, Aspects of Berry phase in QFT, JHEP 04 (2017) 062 [arXiv: 1701.05587] [INSPIRE].

[14] V. Niarchos, Geometry of Higgs-branch superconformal primary bundles, Phys. Rev. D 98 (2018) 065012 [arXiv:1807.04296] [INSPIRE]. 
[15] S. Cecotti and C. Vafa, Topological antitopological fusion, Nucl. Phys. B 367 (1991) 359 [INSPIRE].

[16] S. Gukov and E. Witten, Gauge theory, ramification, and the geometric Langlands program, hep-th/0612073 [INSPIRE].

[17] F.A. Dolan and H. Osborn, On short and semi-short representations for four-dimensional superconformal symmetry, Annals Phys. 307 (2003) 41 [hep-th/0209056] [INSPIRE].

[18] D. Huybrechts, Complex geometry - An introduction, Universitext, Springer, Germany (2005). 